\title{
Statistics-dependent quantum co-walking of two particles in one-dimensional lattices with nearest-neighbor interactions
}

\author{
Xizhou Qin (秦锡洲), ${ }^{1}$ Yongguan Ke (柯勇贯), ${ }^{1}$ Xiwen Guan (管习文) ${ }^{2,3}$ Zhibing Li (李志兵), ${ }^{1}$ \\ Natan Andrei, ${ }^{4}$ and Chaohong Lee (李朝红) ${ }^{1, *}$ \\ ${ }^{1}$ State Key Laboratory of Optoelectronic Materials and Technologies, School of Physics and Engineering, Sun Yat-Sen University, \\ Guangzhou 510275, China \\ ${ }^{2}$ State Key Laboratory of Magnetic Resonance and Atomic and Molecular Physics, Wuhan Institute of Physics and Mathematics, \\ Chinese Academy of Sciences, Wuhan 430071, China \\ ${ }^{3}$ Department of Theoretical Physics, Research School of Physics and Engineering, Australian National University, Canberra, \\ Australian Capital Territory 0200, Australia \\ ${ }^{4}$ Department of Physics, Rutgers University, Piscataway, New Jersey 08854, USA
}

(Received 13 February 2014; revised manuscript received 1 September 2014; published 1 December 2014)

\begin{abstract}
We investigate continuous-time quantum walks of two indistinguishable particles [two bosons, or two fermions, or two hard-core bosons (HCBs)] in one-dimensional lattices with nearest-neighbor interactions. The results for two HCBs are consistent with the recent experimental observation of two-magnon dynamics [Fukuhara et al., Nature (London) 502, 76 (2013)]. The two interacting particles can undergo independent walking and/or co-walking depending on both quantum statistics and interaction strength. Two strongly interacting particles may form a bound state and then co-walk like a single composite particle with a statistics-dependent walk speed. Analytical solutions for the scattering and bound states, which appear in the two-particle quantum walks, are obtained by solving the eigenvalue problem in the two-particle Hilbert space. In the context of degenerate perturbation theory, an effective single-particle model for the quantum co-walking is analytically derived and the walk speed of bosons is found to be exactly three times that of fermions and HCBs. Our result paves the way for experimentally exploring quantum statistics via two-particle quantum walks.
\end{abstract}

DOI: 10.1103/PhysRevA.90.062301

\section{INTRODUCTION}

The quantum walk (QW) [1,2], the quantum counterpart of the classical random walk (CRW), is not only a fundamental phenomenon in quantum transport, but also a practical tool for developing quantum algorithms and implementing quantum computations. In contrast to CRWs, which gradually approach an equilibrium distribution, QWs spread ballistically if there is no decoherence. The nonclassical features of QWs offer versatile applications in quantum simulation [3], quantum computation $[4,5]$, detecting topological states [6-8], probing bound states $[7,9,10]$, and so on.

Up to now, single-particle QWs have been implemented with several experimental systems. In those experiments, the roles of quantum walkers are taken by single particles such as neutral atoms [11], atomic ions [12], photons [13], atomic spin impurities [14], and nuclear-magnetic-resonance systems [15]. Attributable to their superpositions and interference features, single-particle QWs yield an exponential speedup over CRWs [16]. However, it has been demonstrated that such an exponential speedup can be also achieved by classical waves [17].

In contrast, multiparticle QWs may have exotic nonclassical correlations, which may bring new benefits to practical quantum technologies. It has been found that two-particle discrete QWs sensitively depend on the entanglement or correlations $[18,19]$. Naturally, the quantum statistical nature of two bosonic (fermionic) walkers result in the emergence of bunching (anti-bunching) in two-particle QWs [18]. Moreover,

*Corresponding author: chleecn@gmail.com
PACS number(s): 03.67.-a, 05.60.Gg, 42.50.-p, 42.82.Et multiparticle QWs can be used to implement universal quantum computations [5]. By using linear [20-22] and nonlinear photonic waveguide arrays $[23,24]$, two-particle QWs have been implemented in several laboratories. Exotic quantum correlations have been observed even in the absence of interparticle interactions $[22,25,26]$. Recently, the coexistence of free and bound states [27] has been observed via two-particle QWs of atomic spin impurities in one-dimensional (1D) optical lattices [10].

Although there are some studies on two-particle QWs involving quantum statistics and interparticle interactions, most of them only consider either how quantum statistics affects the QWs of two noninteracting particles [21,26] or how interparticle interaction affects the QWs of two interacting particles with a specific quantum statistics $[10,24]$. A comprehensive study on how two-particle QWs depend on both quantum statistics and interparticle interactions is still lacking. It is particularly interesting how the quantum co-walking of two interacting particles quantitatively depends on the quantum statistics of two walkers. Here co-walking means that the two walkers are fully synchronized and walk as a single composite unity.

In this article we investigate two-particle continuous-time QWs in 1D lattices with nearest-neighbor interactions. We concentrate on analyzing quantum statistic affects in the QWs of two interacting particles. We show the bunching and antibunching dynamics induced by the Bose and Fermi natures of quantum walkers and systematically investigate the statistics-dependent quantum co-walking. In addition to the numerical results, we derive an analytical model for the statistics-dependent quantum co-walking by employing degenerate perturbation theory. We present both analytical 
and numerical results, which are consistent with each other. Our analytical results give a quantitative understanding of the quantum statistic effects in the quantum co-walking of two interacting indistinguishable particles. In particular, our prediction on two hard-core bosonic walkers agrees with the experimental observation of the two-magnon dynamics [10]. In the scenario of the quantum-optical analog [28,29], our two-particle QWs can be experimentally verified by the light propagations in 2D waveguide arrays $[29,30]$.

The structure of this paper is as follows. In this section we introduce background and motivation. In Sec. II we describe our models and discuss some key properties of them. In Sec. III we solve the eigenvalue problem in the two-particle Hilbert space and derive several analytical solutions for the two-particle eigenstates and their eigenenergies. In Sec. IV we analyze the two-particle QWs under three different types of statistics: bosonic, fermionic, and hard-core bosonic ones. In both position and momentum spaces, two-body correlations of bosonic and fermionic walkers show subtle bunching and antibunching signatures, respectively. However, hard-core bosonic walkers show an antibunching signature in the position space and a bunching signature in the momentum space. In Sec. V we analytically derive the effective single-particle model for the co-walking of two quantum walkers under strong interparticle interactions. We discuss the implementation of our model and summarize the results in Sec. VI.

\section{MODEL}

We consider QWs of two indistinguishable particles in a 1D lattice system described by the following Hamiltonian with periodic boundary conditions (PBCs):

$$
\hat{H}=-J \sum_{l=-L}^{L}\left(\hat{a}_{l}^{\dagger} \hat{a}_{l+1}+\text { H.c. }\right)+V \sum_{l=-L}^{L} \hat{n}_{l} \hat{n}_{l+1} .
$$

Here the total number of lattice sites is $L_{t}=2 L+1, \hat{a}_{l}^{\dagger}$ $\left(\hat{a}_{l}\right)$ creates (annihilates) a particle on the $l$ th lattice $(l=$ $-L, \ldots, 0, \ldots, L), \hat{n}_{l}=\hat{a}_{l}^{\dagger} \hat{a}_{l}$ is the particle number, $J$ is the nearest-neighbor hopping, and $V$ stands for the nearestneighbor interaction. Below we only discuss the Hamiltonian of the attractive interaction $V<0$.

The two-particle (i.e., $\hat{N}=\sum_{l=-L}^{L} \hat{n}_{l}=2$ ) propagation in our systems represents a class of continuous-time two-particle QWs. The continuous-time QWs can be generalized from the continuous-time CRWs $[2,16]$. A CRW on a graph is described by a matrix $\mathbf{M}$, which transforms the probability distribution (PD) $\mathbf{p}=\left\{p_{l}\right\}$ over a vertex set $\mathbf{v}=\{l\}$ (here $p_{l}$ is the probability of finding the walker at the $l$ th vertex). The elements $\mathbf{M}_{l l^{\prime}}$ give the jumping rate from the $l$ th vertex to the $l^{\prime}$ th vortex. The PD evolution of such a walk follows $\frac{d}{d t} \mathbf{p}(t)=-\mathbf{M} \mathbf{p}(t)$ and the solution is given as $\mathbf{p}(t)=e^{-\mathbf{M} t} \mathbf{p}(0)$ with the initial condition $\mathbf{p}(0)$. In the quantum case, the matrix $\mathbf{M}$ is replaced by the so-called adjacency matrix $\mathbf{H}[4,31]$, which generates an unitary evolution $e^{-i \mathbf{H} t}$ instead of $e^{-\mathbf{M} t}$. Starting from an initial state $\left|\psi_{\text {ini }}\right\rangle$, the quantum state evolves according to $|\psi(t)\rangle=e^{-i \mathbf{H} t}\left|\psi_{\text {ini }}\right\rangle$ and the PD over the vortex set is given by the quantum projection $p_{l}=|\langle l \mid \psi(t)\rangle|^{2}$ with $|l\rangle$ denoting the quantum state of the walker localized at the $l$ th vortex. In our system, the graph for the two walkers is the 1D lattice and the Hamiltonian matrix $\hat{H}$ plays the role of the adjacency matrix.

We consider three typical types of commutation relations (CRs): bosonic, fermionic, and hard-core bosonic ones. The bosonic CRs read $\left[\hat{a}_{l}, \hat{a}_{k}\right]=\left[\hat{a}_{l}^{\dagger}, \hat{a}_{k}^{\dagger}\right]=0$ and $\left[\hat{a}_{l}, \hat{a}_{k}^{\dagger}\right]=\delta_{l k}$. The fermionic CRs obey $\left\{\hat{a}_{l}, \hat{a}_{k}\right\}=\left\{\hat{a}_{l}^{\dagger}, \hat{a}_{k}^{\dagger}\right\}=0$ and $\left\{\hat{a}_{l}, \hat{a}_{k}^{\dagger}\right\}=$ $\delta_{l k}$. The hard-core bosonic CRs are described by $\left[\hat{a}_{l}, \hat{a}_{k}\right]=$ $\left[\hat{a}_{l}^{\dagger}, \hat{a}_{k}^{\dagger}\right]=\left[\hat{a}_{l}, \hat{a}_{k}^{\dagger}\right]=0$ for $l \neq k$, while $\left\{\hat{a}_{l}, \hat{a}_{l}\right\}=\left\{\hat{a}_{l}^{\dagger}, \hat{a}_{l}^{\dagger}\right\}=0$ and $\left\{\hat{a}_{l}, \hat{a}_{l}^{\dagger}\right\}=1$.

The Hamiltonian (1) is associated with the quasiparticle representation for an $X X Z$ Heisenberg chain [32,33]. By using the mapping $|\downarrow\rangle \leftrightarrow|0\rangle,|\uparrow\rangle \leftrightarrow|1\rangle, \hat{S}_{l}^{+} \leftrightarrow \hat{a}_{l}^{\dagger}, \hat{S}_{l}^{-} \leftrightarrow \hat{a}_{l}$, and $\hat{S}_{l}^{z} \leftrightarrow \hat{n}_{l}-\frac{1}{2}$, the hard-core bosonic system is equivalent to the $X X Z$ Heisenberg chain [32]

$$
\begin{aligned}
\hat{H}_{X X Z}= & -J_{\mathrm{ex}} \sum_{l}\left(\hat{S}_{l}^{x} \hat{S}_{l+1}^{x}+\hat{S}_{l}^{y} \hat{S}_{l+1}^{y}+\Delta \hat{S}_{l}^{z} \hat{S}_{l+1}^{z}\right) \\
& +h_{z} \sum_{l} \hat{S}_{l}^{z},
\end{aligned}
$$

with $J_{\mathrm{ex}}=2 J, \Delta=-\frac{V}{2 J}, h_{z}=V$, and $\hat{S}_{l}^{ \pm}=\hat{S}_{l}^{x} \pm i \hat{S}_{l}^{y}$. It has been demonstrated that such an $X X Z$ Heisenberg chain can be realized by ultracold two-level atoms in optical lattices $[10,14,34]$.

\section{TWO-PARTICLE EIGENSTATES}

In this section we solve the eigenvalue problem in the twoparticle Hilbert space and give the eigenstates that appear in the two-particle QWs. Since $[\hat{N}, \hat{H}]=0$, the total particle number $\hat{N}$ is conserved and all initial two-particle states keep evolving in the two-particle Hilbert space. For two bosons, the Hilbert space is spanned by the basis

$$
\mathcal{B}_{B}^{(2)}=\left\{\left|l_{1} l_{2}\right\rangle=\left(1+\delta_{l_{1} l_{2}}\right)^{-1 / 2} \hat{a}_{l_{1}}^{\dagger} \hat{a}_{l_{2}}^{\dagger}|\mathbf{0}\rangle\right\},
$$

with $-L \leqslant l_{1} \leqslant l_{2} \leqslant L$. For two fermions or two hard-core bosons (HCBs), the Hilbert spaces are spanned by the basis

$$
\mathcal{B}_{F H}^{(2)}=\left\{\left|l_{1} l_{2}\right\rangle=\hat{a}_{l_{1}}^{\dagger} \hat{a}_{l_{2}}^{\dagger}|\mathbf{0}\rangle\right\},
$$

with $-L \leqslant l_{1}<l_{2} \leqslant L$. Given $\mathcal{B}_{B}^{(2)}$ and $\mathcal{B}_{F H}^{(2)}$, it is easy to find the Hamiltonian matrix $H^{(2)}$ in the two-particle sector.

Introducing $C_{l_{1} l_{2}}=\left\langle\mathbf{0}\left|\hat{a}_{l_{2}} \hat{a}_{l_{1}}\right| \Psi\right\rangle$, the eigenstates can be expanded as $|\Psi\rangle=\sum_{l_{1} \leqslant l_{2}} \psi_{l_{1} l_{2}}\left|l_{1} l_{2}\right\rangle$ with $\psi_{l_{1} l_{2}}=C_{l_{1} l_{2}}(1+$ $\left.\delta_{l_{1} l_{2}}\right)^{-1 / 2}$. Independent of the quantum statistics of particles, the eigenequation $\hat{H}|\Psi\rangle=E|\Psi\rangle$ can be written in the unified form

$$
\begin{aligned}
E C_{l_{1} l_{2}}= & -J\left(C_{l_{1}, l_{2}+1}+C_{l_{1}, l_{2}-1}+C_{l_{1}+1, l_{2}}+C_{l_{1}-1, l_{2}}\right) \\
& +V \delta_{l_{1}, l_{2} \pm 1} C_{l_{1} l_{2}},
\end{aligned}
$$

with $\delta_{l_{1}, l_{2} \pm 1}=1$ if $l_{1}=l_{2} \pm 1$ and $\delta_{l_{1}, l_{2} \pm 1}=0$ if $l_{1} \neq l_{2} \pm 1$. Here the PBC requires $C_{l_{1}+L_{t}, l_{2}}=C_{l_{1}, l_{2}+L_{t}}=C_{l_{1} l_{2}}$. The CRs require that $C_{l_{1} l_{2}}=C_{l_{2} l_{1}}$ for bosons, $C_{l_{1} l_{1}}=0$ and $C_{l_{1} l_{2}}=$ $-C_{l_{2} l_{1}}$ for fermions, and $C_{l_{1} l_{1}}=0$ and $C_{l_{1} l_{2}}=C_{l_{2} l_{1}}$ for HCBs.

The motion of the two-particle system can be separated by the motion of the center of mass $R=\frac{1}{2}\left(l_{1}+l_{2}\right)$ and that of the relative position $r=l_{1}-l_{2}$. By employing the ansatz $C_{l_{1} l_{2}}=e^{i K R} \phi(r)$, the eigenequation reads

$$
E \phi(r)=J_{K}[\phi(r-1)+\phi(r+1)]+V \delta_{r, \pm 1} \phi(r),
$$


with $J_{K}=-2 J \cos \left(\frac{K}{2}\right), \delta_{r, \pm 1}=1$ if $r= \pm 1$, and $\delta_{r, \pm 1}=$ 0 if $r \neq \pm 1$. Therefore, the $\mathrm{PBC}$ requires $e^{i K L_{t}}=1$ and $\phi\left(r+L_{t}\right)=e^{i K L_{t} / 2} \phi(r)$ with the quantized total quasimomentum $K=2 \pi \alpha / L_{t}$ with $\alpha=-L,-L+1, \ldots, L$. Correspondingly, the CRs require that $\phi(r)=\phi(-r)$ for bosons, $\phi(0)=0$ and $\phi(r)=-\phi(-r)$ for fermions, $\phi(0)=0$ and $\phi(r)=\phi(-r)$ for HCBs.

The PBC and CRs indicate that $\{\phi(r) \mid r=0, \ldots, L\}$ for bosons and $\{\phi(r) \mid r=1, \ldots, L\}$ for fermions or HCBs are independent variables. Thus the two-particle Hamiltonian matrix block for bosons with total quasimomentum $K$ can be written as

$$
\hat{H}_{B}^{(2)}(K)=\left(\begin{array}{cccccc}
0 & 2 J_{K} & & & & \\
J_{K} & V & J_{K} & & & \\
& J_{K} & 0 & J_{K} & & \\
& & \ddots & \ddots & \ddots & \\
& & & J_{K} & 0 & J_{K} \\
& & & & J_{K} & J_{K}^{B}
\end{array}\right) ;
$$

the one for fermions reads

$$
\hat{H}_{F}^{(2)}(K)=\left(\begin{array}{ccccc}
V & J_{K} & & & \\
J_{K} & 0 & J_{K} & & \\
& \ddots & \ddots & \ddots & \\
& & J_{K} & 0 & J_{K} \\
& & & J_{K} & J_{K}^{F}
\end{array}\right)
$$

and the one for HCBs is in the form

$$
\hat{H}_{H}^{(2)}(K)=\left(\begin{array}{ccccc}
V & J_{K} & & & \\
J_{K} & 0 & J_{K} & & \\
& \ddots & \ddots & \ddots & \\
& & J_{K} & 0 & J_{K} \\
& & & J_{K} & J_{K}^{H}
\end{array}\right) .
$$

Here we define $J_{K}^{H}=J_{K}^{B}=-J_{K}^{F}=e^{i K L_{t} / 2} J_{K}$.

The Hamiltonian matrices (5)-(7) can be diagonalized numerically and analytically [35]. When $V \neq 0$, for all three cases (bosons, fermions, and HCBs), there are two types of eigenstates: bound states (BSs) and scattering states (SSs). For SSs, the amplitude of the wave function $\phi(r)$ oscillates as the relative position $r$, while for BSs, it decays exponentially. The general eigenstate for Eq. (4) can be expressed as

$$
\phi(r)=A_{+} e^{i k r}+A_{-} e^{-i k r}
$$

with the two constants $\left(A_{+}, A_{-}\right)$and the quasimomentum $k$. For SSs, the quasimomentum $k$ is real. However, for BSs, the quasimomentum $k$ is purely imaginary. The eigenenergy is given as

$$
E_{K, k}^{(2)}=2 J_{K} \cos (k)=-4 J \cos \left(\frac{K}{2}\right) \cos (k),
$$

with the quasimomentum $k$ determined by the physical parameters and the statistical properties. Below we will show how to determine the quasimomentum $k$.

\section{A. Scattering states}

Due to the real value of $k$, the scattering states are invariant under the transformation $k \rightarrow k \pm 2 \pi$ and $k \rightarrow-k$. Thus we only need to consider $0 \leqslant k<\pi$.
For fermions, the $\mathrm{PBC}$ and $\mathrm{CRs}$ require that

$$
\begin{aligned}
\left(J_{K}-V e^{i k}\right) A_{+}+\left(J_{K}-V e^{-i k}\right) A_{-} & =0, \\
e^{i k L_{t}} A_{+}+(-1)^{\alpha} A_{-} & =0 .
\end{aligned}
$$

By eliminating $A_{+}$and $A_{-}$, one can obtain that the quasimomentum $k$ obeys

$$
e^{i k L_{t}}=(-1)^{\alpha} \frac{J_{K}-V e^{i k}}{J_{K}-V e^{-i k}} .
$$

To give all possible values of $k$, one has to solve Eq. (10), which is actually an algebraic equation of $e^{i k}$. Thus, the corresponding eigenstate reads

$$
\phi(r) \propto e^{i k r}-e^{-i k r} e^{i(K / 2+k) L_{t}}, \quad 1 \leqslant r \leqslant N .
$$

For HCBs, the quasimomentum $k$ satisfies

$$
e^{i k L_{t}}=(-1)^{\alpha-1} \frac{J_{K}-V e^{i k}}{J_{K}-V e^{-i k}}
$$

and the corresponding eigenstate is

$$
\phi(r) \propto e^{i k r}+e^{-i k r} e^{i(K / 2+k) L_{t}}, \quad 1 \leqslant r \leqslant N .
$$

For bosons, the ansatz should be modified as

$$
\phi(r)= \begin{cases}\phi_{0}, & r=0 \\ A_{+} e^{i k r}+A_{-} e^{-i k r}, & 1 \leqslant r \leqslant N,\end{cases}
$$

with the quasimomentum $k$ satisfying

$$
e^{i k L_{t}}=(-1)^{\alpha} \frac{J_{K}\left(e^{i k}-e^{-i k}\right)+V\left(1+e^{2 i k}\right)}{J_{K}\left(e^{i k}-e^{-i k}\right)-V\left(1+e^{-2 i k}\right)} .
$$

Thus the corresponding eigenstate is given as

$$
\phi(r) \propto e^{i k r}+e^{-i k r} e^{i(K / 2+k) L_{t}}, \quad 1 \leqslant r \leqslant N,
$$

with $\phi(0)=\phi_{0}=\phi(1) / \cos (k)$.

\section{B. Bound states}

The bound states correspond to purely imaginary $k=i \eta$ $(\eta>0)$ satisfying the conditions (10), (12), and (15). For a finite $L_{t}$, no compact formulas for $\eta$ are available. However, when $L_{t}$ is sufficiently large, the factor $e^{i k L_{t}}=e^{-\eta L_{t}}$ become small and, as an approximation, one can assume $e^{-\eta L_{t}} \approx 0$, which is exact $\left(e^{-\eta L_{t}}=0\right)$ when $L_{t} \rightarrow \infty$. Then the conditions for $\eta \mathrm{read}$

$$
\begin{aligned}
& J_{K}=V e^{-\eta} \text { for fermions or HCBs, } \\
& J_{K}\left(e^{-\eta}-e^{\eta}\right)+V\left(1+e^{-2 \eta}\right)=0 \text { for bosons. }
\end{aligned}
$$

Solving Eqs. (17), one can obtain

$$
e^{\eta}=V / J_{K}
$$

for fermions or HCBs (as long as $|V|>\left|J_{K}\right|$ ) and

$$
\begin{aligned}
e^{\eta} & =\frac{1}{3}\left(\beta+\frac{3+\beta^{2}}{\Delta_{0}}+\Delta_{0}\right), \\
\Delta_{0} & =\left(18 \beta+\beta^{3}+3 \sqrt{3} \sqrt{\beta^{4}+11 \beta^{2}-1}\right)^{1 / 3}
\end{aligned}
$$

for bosons (as long as $\beta^{2}\left(\beta^{2}+11\right)>1$ ), where $\beta=V / J_{K}$.

According to Eq. (9), given $k=i \eta$ for a BS, its eigenenergy reads

$$
E_{K, \eta}^{(2)}=2 J_{K} \cosh (\eta)
$$



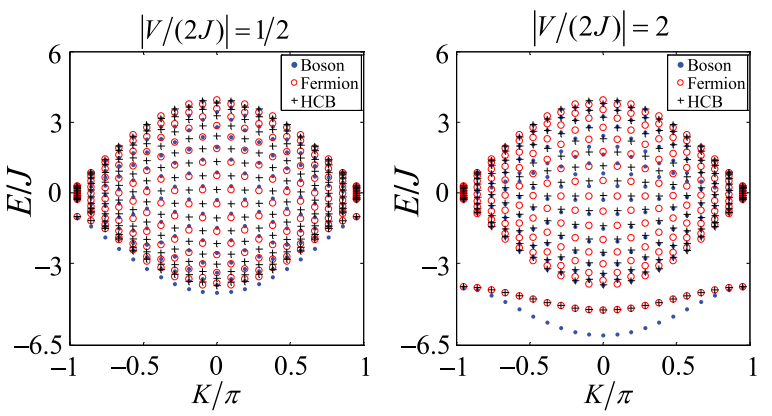

FIG. 1. (Color online) Two-particle spectrum for a 21-site system (1) with different interaction strength $|V / 2 J|$. Shown on the left is the weak interaction $|V / 2 J|=1 / 2$ and on the right is the strong interaction $|V / 2 J|=2$. Each point represents an eigenenergy $E$ for a given quasimomentum $K$.

with $\eta$ determined by Eq. (17). Thus, for the BS of fermions or HCBs, according to Eq. (18), its eigenenergy reads

$$
E_{F H}^{(2)}(K)=V+\frac{4 J^{2}}{V} \cos ^{2}\left(\frac{K}{2}\right)
$$

when $|V / 2 J|>1$ and $L_{t} \rightarrow \infty$. Obviously, Eq. (21) fully agrees with the eigenenergies obtained from the Bethe ansatz [36]. For the case of strongly interacting bosons, that is, $|\beta| \rightarrow$ $\infty$, we have $e^{\eta}>\beta=V / J_{K}$ and $e^{\eta} / \beta \rightarrow 1$ for positive $\beta$. Thus, according to Eqs. (18)-(20), for the case of an attractive interaction, the BS eigenenergy of bosons is lower than that of fermions or HCBs and their difference vanishes when $|V / 2 J| \rightarrow \infty$.

In Fig. 1 we show the energy spectrum for the two-particle system. For a weak interaction $|V / 2 J|<1$, there is only one band, in which SSs and BSs are mixed. For a strong interaction $|V / 2 J|>1$, there are two minibands, in which the upper band corresponds to SSs and the lower band corresponds to BSs.

\section{TWO-PARTICLE QUANTUM WALKS}

In this section we focus on the time-evolution dynamics of two-particle states, i.e., the two-particle QWs. In particular, by analyzing two-particle correlations in both position and momentum spaces, we explore how the interaction and statistics affect the two-particle QWs.

In units of $\hbar=1$, the two-particle QWs obeys the timedependent Schrödinger equation

$$
i \frac{d}{d t}|\psi(t)\rangle=H^{(2)}|\psi(t)\rangle,
$$

with $|\psi(t)\rangle=\sum_{l_{1} \leqslant l_{2}} \psi_{l_{1} l_{2}}(t)\left|l_{1} l_{2}\right\rangle$ for bosons and $|\psi(t)\rangle=$ $\sum_{l_{1}<l_{2}} \psi_{l_{1} l_{2}}(t)\left|l_{1} l_{2}\right\rangle$ for fermions and HCBs. Here we consider the two-particle QWs from an initial state of two particles sitting in neighboring lattice sites $\left|\psi_{\text {ini }}\right\rangle=\hat{a}_{0}^{\dagger} \hat{a}_{1}^{\dagger}|\mathbf{0}\rangle$. Here $|\mathbf{0}\rangle$ denotes the vacuum state.

To explore the correlation between two quantum walkers, we calculate the time-dependent two-particle correlation in position space

$$
\Gamma_{q r}(t)=\left\langle\psi(t)\left|\hat{a}_{q}^{\dagger} \hat{a}_{r}^{\dagger} \hat{a}_{r} \hat{a}_{q}\right| \psi(t)\right\rangle
$$

and those in momentum space

$$
\Gamma_{\alpha \beta}(t)=\left\langle\psi(t)\left|\hat{c}_{\alpha}^{\dagger} \hat{c}_{\beta}^{\dagger} \hat{c}_{\beta} \hat{c}_{\alpha}\right| \psi(t)\right\rangle,
$$

with $|\psi(t)\rangle$ in Eq. (22). Here $\hat{c}_{\alpha}^{\dagger}=\frac{1}{\sqrt{L_{t}}} \sum_{l=-L}^{L} e^{-i p_{\alpha} l} \hat{a}_{l}^{\dagger}$ is the discrete Fourier transformation of $\hat{a}_{l}^{\dagger}$, in which the quasimomentum $p_{\alpha}=2 \pi \alpha / L_{t}$ and the integer $\alpha=-L$, $-L+1, \ldots, L$. The two-particle correlation in position and momentum spaces for different quantum statistics and interaction strength provide clear insight into the two-particle QWs (see Figs. 2 and 3 for a 21-site system). For systems with the same parameters but different lattice sizes $L_{t}$, our numerical results show that, before the two particles collide with the boundaries, the finite-size effect and the boundary effect are negligible.

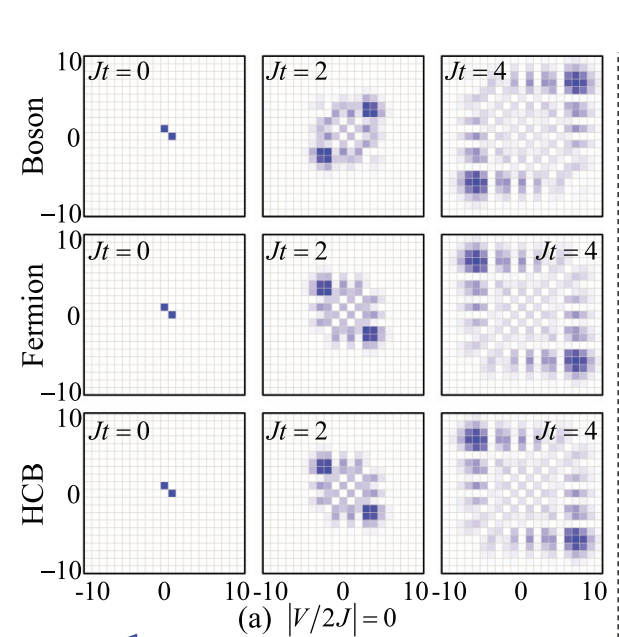

independent walking

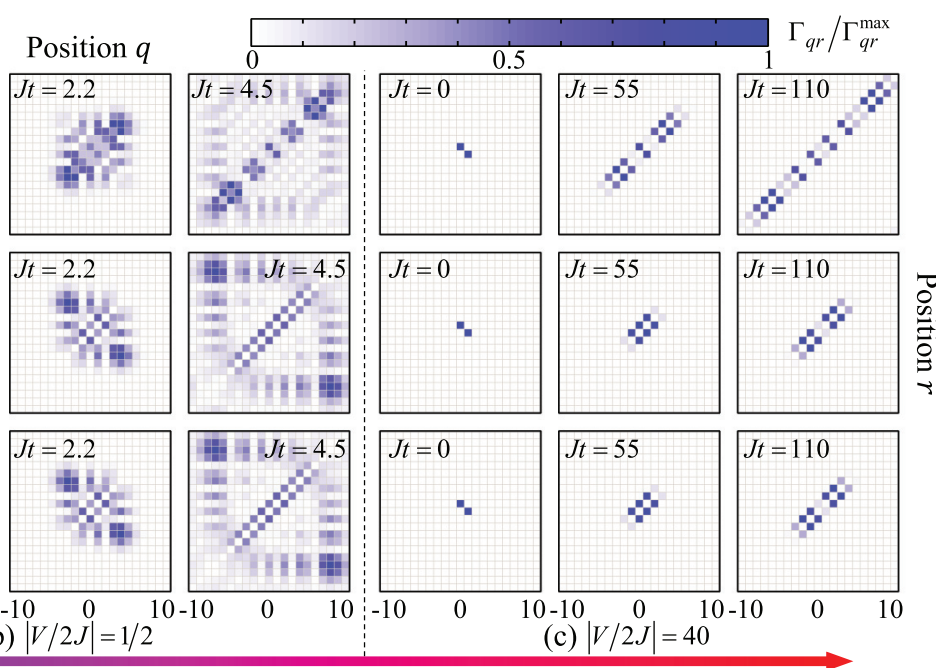

co-walking

FIG. 2. (Color online) Two-particle correlations of quantum walkers in position space. The first, second, and third rows correspond to Bose, Fermi, and HCB statistics, respectively. The interaction-hopping ratios $|V / 2 J|$ are (a) 0 , (b) 0.5 , and (c) 40. Here the total number of lattice sites is $L_{t}=21$, the evolution time is given by $J t$, and we only show the instantaneous correlations before the particles collide with the boundaries $l= \pm 10$. 

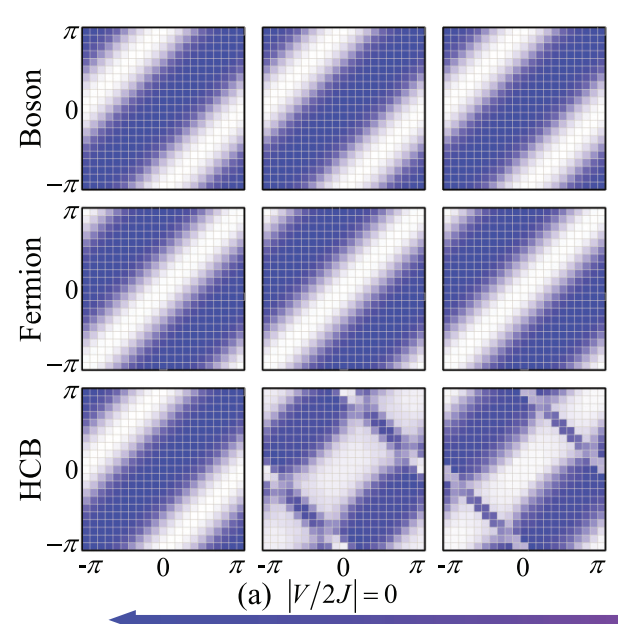
independent walking

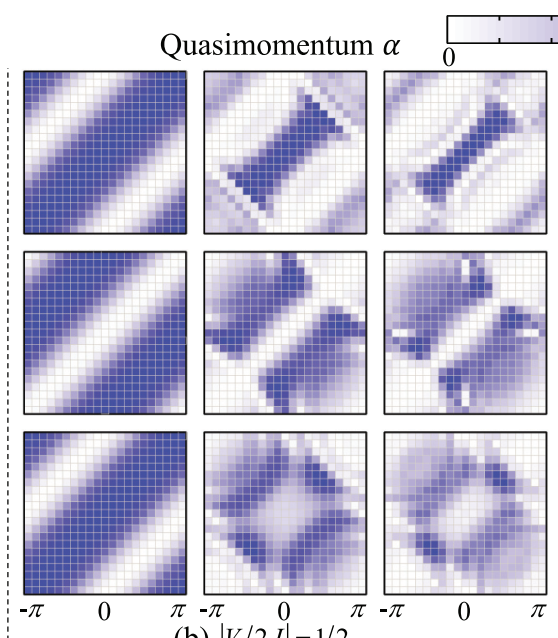

(b) $|V / 2 J|=1 / 2$

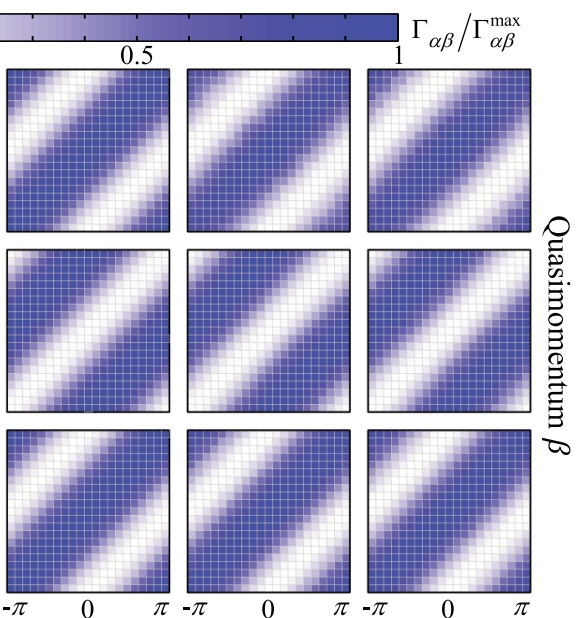

(c) $|V / 2 J|=40$

FIG. 3. (Color online) Two-particle correlations of quantum walkers in momentum space with the same setting as in Fig. 2.

It is possible to distinguish co-walking from independent walking through examining the evolution of the spatial correlation $\Gamma_{q r}(t)$. The significant correlations at two specific lines $(q=r \pm d)$ in the $(q, r)$ plane is a signature of the co-walking of the two particles, where $d$ is a fixed integer dependent on the form of the interparticle interaction. For our systems with the nearest-neighbor interactions, the spatial correlation peaks [i.e., the peaks of $\Gamma_{q r}(t)$ ] appear on the two minor-diagonal lines $(q=r \pm 1)$. This is a typical signature of the two-particle co-walking.

In position space, the correlations of two bosonic walkers (the first row of Fig. 2) show bunching behavior, while the correlations of two fermionic walkers (the second row of Fig. 2) and two hard-core bosonic walkers (the third row of Fig. 2) show antibunching behavior. We observe that the correlations of fermions and HCBs in position space show almost no difference. This is because a spin- $\frac{1}{2}$ Heisenberg $X X Z$ model, which is equivalent to a hard-core Bose-Hubbard model [32], can be exactly mapped onto a Hubbard-like model of spinless fermions via Jordan-Wigner transformation [33]. Although boundary conditions for the Hubbard-like model of spinless fermions depend on the total particle number [33], before the two walkers hit the boundaries, the boundary condition effect on the two fermionic walkers is the same as that on the two hard-core bosonic walkers. Therefore, the correlations are almost the same for fermions and HCBs in position space.

On the other hand, the correlations of bosonic and hard-core bosonic walkers in momentum space show bunching behavior (see the first and third rows of Fig. 3). Nevertheless, the correlations of fermionic walkers (the second row of Fig. 3) show antibunching behavior. This means that bunching and antibunching in momentum space can show the difference between fermions and HCBs. Therefore, bunching and antibunching of the two quantum walkers in both position and momentum spaces completely reveal the difference among bosons, fermions, and HCBs.

The spatial correlations $\Gamma_{q r}$ on the minor diagonal lines $(q=r \pm 1)$ are gradually enhanced when the interactionhopping ratio increases (see Fig. 2). Since $\Gamma_{q, q \pm 1}$ presents a joint probability of finding one walker on the $q$ th site and the other walker on the $(q \pm 1)$ th site, the significant correlations on the minor diagonal lines is a robust signature of quantum co-walking. The quantum co-walking is also an important signature of the existence of two-particle bound states (see $[10,27]$ for the case of two magnons). A detailed discussion of quantum co-walking will be presented in the next section. Usually, two interacting quantum walkers simultaneously undergo independent walking and co-walking when the interaction is not strong enough.

\section{EFFECTIVE DYNAMICS OF TWO-PARTICLE QUANTUM CO-WALKING}

In this section we will analytically derive an effective single-particle model for the quantum co-walking of two interacting particles and discuss the statistics-dependent behavior of quantum co-walking. We present a quantitative description of the quantum statistics effect in two-particle QWs.

Under strong interparticle interactions $(|V / J| \gg 1)$, the two quantum walkers behave as a single composite particle and their QWs are dominated by quantum co-walking. As $|V / J| \gg 1$, one thus can treat the hopping term

$$
\hat{H}_{1}=-J \sum_{l=-L}^{L}\left(\hat{a}_{l}^{\dagger} \hat{a}_{l+1}+\text { H.c. }\right)
$$

as a perturbation to the interaction term

$$
\hat{H}_{0}=V \sum_{l=-L}^{L} \hat{n}_{l} \hat{n}_{l+1}
$$

in the considered Hamiltonian (1). By employing the secondorder perturbation theory for degenerate systems [37], we analytically obtain an effective single-particle model for the co-walking of the two quantum walkers.

To implement the perturbation analysis, we should give the projection operator onto the subspace involved the quantum co-walking and the projection operator onto the orthogonal component of the involved subspace. The 
unperturbed Hamiltonian $\hat{H}_{0}$ has only two eigenvalues: (i) $E_{0}=V(<0)$ for the $L_{t}$-fold degenerated ground states $\left\{\left|G_{l}\right\rangle=|l, l+1\rangle:-L \leqslant l \leqslant L\right\}$ and (ii) $E_{l_{1} l_{2}}=0$ for excited eigenstates $\left\{\left|E_{l_{1} l_{2}}\right\rangle=\left|l_{1}, l_{2}\right\rangle: l_{1} \neq l_{2} \pm 1,-L \leqslant l_{1} \leqslant l_{2} \leqslant L\right\}$ for bosons and $\left\{\left|E_{l_{1} l_{2}}\right\rangle=\left|l_{1}, l_{2}\right\rangle: l_{1} \neq l_{2} \pm 1,-L \leqslant l_{1}<\right.$ $\left.l_{2} \leqslant L\right\}$ for fermions and HCBs. The quantum co-walking only involves the subspace spanned by $L_{t}$ independent ground states $\left\{\left|G_{l}\right\rangle\right\}$. Defining $\mathcal{U}_{0}=\left\{\left|G_{l}\right\rangle\right\}$, the projection operator onto $\mathcal{U}_{0}$ is

$$
\hat{P}_{0}=\sum_{l}\left|G_{l}\right\rangle\left\langle G_{l}\right| \text {. }
$$

Introducing $\mathcal{V}_{0}$ as the orthogonal complement of $\mathcal{U}_{0}$, the projection onto $\mathcal{V}_{0}$ reads

$$
\hat{S}=\sum_{E_{l_{1} l_{2}} \neq E_{0}} \frac{1}{E_{0}-E_{l_{1} l_{2}}}\left|E_{l_{1} l_{2}}\right\rangle\left\langle E_{l_{1} l_{2}}\right| \text {. }
$$

Therefore, the effective Hamiltonian up to second order is given as

$$
\hat{H}_{\mathrm{eff}}^{(2)}=\hat{h}_{0}+\hat{h}_{2}=E_{0} \hat{P}_{0}+\hat{P}_{0} \hat{H}_{1} \hat{S} \hat{H}_{1} \hat{P}_{0} .
$$

Since $E_{l_{1} l_{2}}=0$, we have

$$
\begin{aligned}
\hat{h}_{2}= & \frac{J^{2}}{V} \sum_{l l^{\prime} j j^{\prime} l_{1} l_{2}}\left[\left|G_{l}\right\rangle\left\langle G_{l}\left|\hat{T}_{j}\right| E_{l_{1} l_{2}}\right\rangle\right. \\
& \left.\times\left\langle E_{l_{1} l_{2}}\left|\hat{T}_{j^{\prime}}\right| G_{l^{\prime}}\right\rangle\left\langle G_{l^{\prime}}\right|\right],
\end{aligned}
$$

where the hopping operators $\hat{T}_{k}=\hat{a}_{k}^{\dagger} \hat{a}_{k+1}+\hat{a}_{k+1}^{\dagger} \hat{a}_{k}$, the summation indices $\left\{l, l^{\prime}, j, j^{\prime}\right\}$ take values from $\{-L,-L+$ $1, \ldots, L\}$, and $\left\{l_{1}, l_{2}\right\}$ is summed over all states of $E_{l_{1} l_{2}}=0$.

Introducing the notation

$$
\begin{gathered}
T_{l_{1} l_{2}}^{j l}=\left\langle G_{l}\left|\left(\hat{a}_{j}^{\dagger} \hat{a}_{j+1}+\hat{a}_{j+1}^{\dagger} \hat{a}_{j}\right)\right| E_{l_{1} l_{2}}\right\rangle, \\
\left|G_{l_{1} l_{2}}^{\prime}\right\rangle=\sum_{j l} T_{l_{1} l_{2}}^{j l}\left|G_{l}\right\rangle,
\end{gathered}
$$

we have $\left\langle G_{l_{1} l_{2}}^{\prime}\right|=\sum_{j^{\prime} l^{\prime}}\left\langle G_{l^{\prime}}\right| T_{l_{1} l_{2}}^{j^{\prime} l^{\prime} *}$ and

$$
\begin{aligned}
\hat{h}_{2} & =\frac{J^{2}}{V} \sum_{l l^{\prime} j j^{\prime} l_{1} l_{2}}\left|G_{l}\right\rangle T_{l_{1} l_{2}}^{j l} T_{l_{1} l_{2}}^{j^{\prime} l^{\prime} *}\left\langle G_{l^{\prime}}\right| \\
& =\frac{J^{2}}{V} \sum_{l_{1} l_{2}}\left|G_{l_{1} l_{2}}^{\prime}\right\rangle\left\langle G_{l_{1} l_{2}}^{\prime}\right| .
\end{aligned}
$$

By using the CRs and $\hat{a}_{l} \hat{a}_{k}^{\dagger}|\mathbf{0}\rangle=\delta_{l k}|\mathbf{0}\rangle$, after some algebra we obtain

$$
\begin{aligned}
\left|G_{l_{1} l_{2}}^{\prime}\right\rangle= & \sqrt{2} \delta_{l_{1} l_{2}}\left(\left|G_{l_{1}-1}\right\rangle+\left|G_{l_{1}}\right\rangle\right)+\delta_{l_{1}, l_{2}-2}\left(\left|G_{l_{1}}\right\rangle+\left|G_{l_{1}+1}\right\rangle\right) \\
& +\epsilon^{\prime} \delta_{l_{1}-2, l_{2}}\left(\left|G_{l_{2}}\right\rangle+\left|G_{l_{2}+1}\right\rangle\right) .
\end{aligned}
$$

Here $\epsilon^{\prime}=1$ for bosons and HCBs, while $\epsilon^{\prime}=-1$ for fermions. Inserting Eq. (32) into Eq. (31), we get

$$
\begin{aligned}
\hat{h}_{2}= & \frac{J^{2}}{V} \sum_{l_{1} l_{2}}\left[2 \delta_{l_{1} l_{2}}\left(\left|G_{l_{1}-1}\right\rangle+\left|G_{l_{1}}\right\rangle\right)\left(\left|G_{l_{1}-1}\right|+\left\langle G_{l_{1}}\right|\right)\right. \\
& +\delta_{l_{1}, l_{2}-2}\left(\left|G_{l_{1}}\right\rangle+\left|G_{l_{1}+1}\right\rangle\right)\left(\left\langle G_{l_{1}}\right|+\left\langle G_{l_{1}+1}\right|\right) \\
& \left.+\delta_{l_{1}-2, l_{2}}\left(\left|G_{l_{2}}\right\rangle+\left|G_{l_{2}+1}\right\rangle\right)\left(\left|G_{l_{2}}\right|+\left\langle G_{l_{2}+1}\right|\right)\right] .
\end{aligned}
$$

For the case of fermions or HCBs, $\delta_{l_{1} l_{2}}=0$ and $\delta_{l_{1}, l_{2}-2}=1$ for $\left(l_{1}, l_{2}\right)=(l, l+2)$ with $l=(-L,-L+1, \ldots, L-2)$ and $\delta_{l_{1}-2, l_{2}}=1$ for $\left(l_{1}, l_{2}\right)=(-L, L-1)$ and $(-L+1, L)$; thus we have

$$
\hat{h}_{2}=\frac{J^{2}}{V} \sum_{q=-L}^{L}\left(\left|G_{q}\right\rangle+\left|G_{q+1}\right\rangle\right)\left(\left\langle G_{q}\right|+\left\langle G_{q+1}\right|\right) .
$$

For the case of bosons, besides the terms included in the case of fermions or HCBs, $\delta_{l_{1} l_{2}}=1$ for $\left(l_{1}, l_{2}\right)=(l, l)$ with $l=(-L$, $-L+1, \ldots, L-1, L)$ should be included; thus we have

$$
\hat{h}_{2}=\frac{3 J^{2}}{V} \sum_{q=-L}^{L}\left(\left|G_{q}\right\rangle+\left|G_{q+1}\right\rangle\right)\left(\left\langle G_{q}\right|+\left\langle G_{q+1}\right|\right) .
$$

In our model of nearest-neighbor interaction, for two walkers starting from two neighbor lattice sites, their co-walking can be described by a superposition of multiple ground states $\left|G_{q}\right\rangle=$ $\hat{a}_{q}^{\dagger} \hat{a}_{q+1}^{\dagger}|\mathbf{0}\rangle=\left|n_{q}=1, n_{q+1}=1\right\rangle$ with different $q$ (where $q=$ $-L,-L+1, \ldots, L-1, L)$. During the process of co-walking, the two particles behave like a single composite particle.

In order to capture the single-particle nature of the cowalking, we introduce creation operators $\hat{b}_{q}^{\dagger}$ for the composite particle consisting of one particle on the $q$ th lattice site and the other particle on the $(q+1)$ th lattice site. Explicitly, $\hat{b}_{q}^{\dagger} \Leftrightarrow \hat{a}_{q}^{\dagger} \hat{a}_{q+1}^{\dagger}$ and $\left|n_{q}^{c}=1\right\rangle=\hat{b}_{q}^{\dagger}|\mathbf{0}\rangle \Leftrightarrow\left|n_{q}=1, n_{q+1}=1\right\rangle=$ $\hat{a}_{q}^{\dagger} \hat{a}_{q+1}^{\dagger}|\mathbf{0}\rangle$. Then, from Eq. (35), the two bosonic walkers obey an effective single-particle Hamiltonian

$$
\hat{H}_{\mathrm{eff}}^{B}=J_{\mathrm{eff}}^{B} \sum_{q}\left(\hat{b}_{q}^{\dagger} \hat{b}_{q+1}+\hat{b}_{q+1}^{\dagger} \hat{b}_{q}\right)+\mu_{\mathrm{eff}}^{B} \sum_{q} \hat{b}_{q}^{\dagger} \hat{b}_{q},
$$

with the hopping strength $J_{\text {eff }}^{B}=\frac{3 J^{2}}{V}$ and the chemical potential $\mu_{\mathrm{eff}}^{B}=V+\frac{6 J^{2}}{V}$. The spectrum of the Hamiltonian (36) can be obtained by substituting the ansatz $|\psi\rangle=\sum_{m} e^{i K m} \hat{b}_{m}^{\dagger}|\mathbf{0}\rangle$ into the eigenvalue problem $\hat{H}_{\text {eff }}^{B}|\psi\rangle=E_{\text {eff }}^{B}|\psi\rangle$. With some analytical calculations, it is easy to yield the single-quasiparticle spectrum

$$
E_{\text {eff }}^{B}(K)=V+\frac{12 J^{2}}{V} \cos ^{2}\left(\frac{K}{2}\right) .
$$

Similarly, from Eq. (34), the two fermionic walkers and the two hard-core bosonic walkers obey the same effective singleparticle Hamiltonian

$$
\hat{H}_{\mathrm{eff}}^{F H}=J_{\mathrm{eff}}^{F H} \sum_{q}\left(\hat{b}_{q}^{\dagger} \hat{b}_{q+1}+\hat{b}_{q+1}^{\dagger} \hat{b}_{q}\right)+\mu_{\mathrm{eff}}^{F H} \sum_{q} \hat{b}_{q}^{\dagger} \hat{b}_{q},
$$

but with $J_{\mathrm{eff}}^{F H}=\frac{J^{2}}{V}, \mu_{\mathrm{eff}}^{F H}=V+\frac{2 J^{2}}{V}$ and spectra

$$
E_{\text {eff }}^{F H}(K)=V+\frac{4 J^{2}}{V} \cos ^{2}\left(\frac{K}{2}\right) .
$$

We observe that, for fixed values of $J$ and $V$, the hopping strengths $J_{\text {eff }}^{B}$ and $J_{\text {eff }}^{F H}$ of the composite particle essentially depend on their quantum statistics. This means that quantum statistics has a significant effect on the co-walking of two interacting walkers. In time-evolution dynamics, different values of hopping strength mean different walk speed. Thus it is possible to explore statistics-dependent quantum co-walking via observing the walk dynamics. 

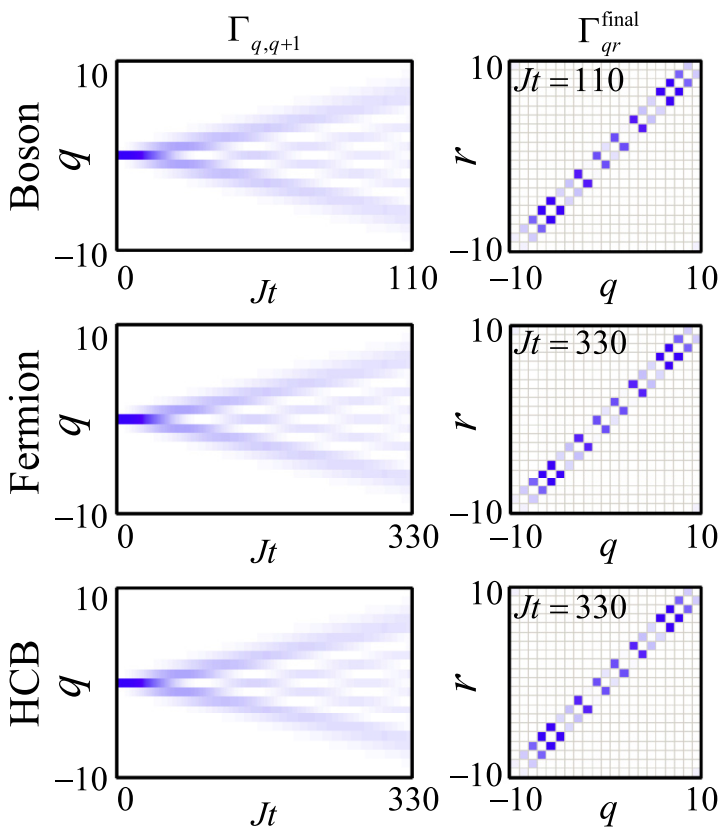

FIG. 4. (Color online) Quantum co-walking of two strongly interacting walkers with $|V / 2 J|=40$. Shown on the left is the time evolution of the minor diagonal correlations $\Gamma_{q, q+1}$ and on the right is the two-particle correlations $\Gamma_{q, r}^{\mathrm{final}}$ for the final states.

In Fig. 4 we show our numerical results for the time evolution of the minor diagonal correlations $\Gamma_{q, q+1}$ and the final two-particle correlations $\Gamma_{q, r}^{\mathrm{final}}$ with $|V / 2 J|=40$. From the correlations $\Gamma_{q, r}^{\text {final }}$ in the right column of Fig. 4, we find that the two strongly interacting walkers are dominated by quantum co-walking. From the time evolution of $\Gamma_{q, q+1}$ in the left column of Fig. 4, we see that the walk speed of two bosonic walkers is just three times that of the two fermionic and hard-core bosonic walkers. These numerical results of spread are consistent with our analytical prediction $J_{\text {eff }}^{B}=3 J_{\text {eff }}^{F H}$ from the second-order perturbation theory.

\section{SUMMARY AND DISCUSSION}

We have explored how quantum statistics and interparticle interactions affect two-particle QWs in 1D lattices with nearest-neighbor interactions. Due to the interparticle interactions, two particles with different quantum statistics undergo independent walking and/or co-walking. The QWs are dominated by independent walking in the weak-interaction limit and by co-walking in the strong-interaction limit. We have analytically derived the effective single-particle model for the co-walking of two strongly interacting particles. We find that the walk speed for the co-walking of two bosons is exactly three times that for the co-walking of two fermions or two HCBs. Although we only consider the two-particle QWs in attractive systems $(V<0)$ in this article, similar QWs may be found in repulsive systems $(V>0)$, which have free scattering states in the lower band and repulsively bound states in the upper band [38]. Our results for the case of two HCBs agree with the recent experimental observation of quantum dynamics of two atomic spin impurities [10]. Besides observing bound states
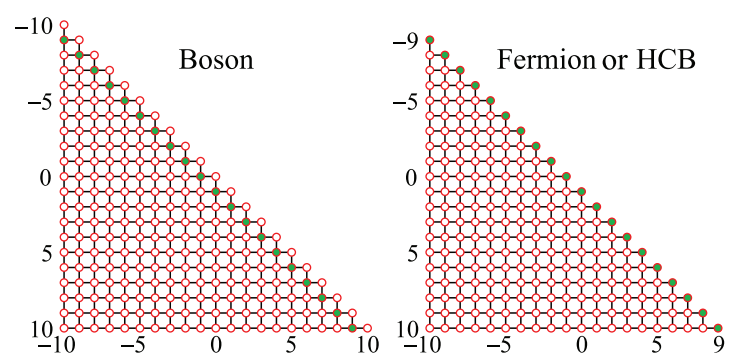

FIG. 5. (Color online) Classical simulation with twodimensional optical waveguide arrays. Each circle represents a waveguide. Green-colored and uncolored circles label waveguides with different refractive indices. The black lines connecting different circles denote their couplings. Shown on the left are the waveguide arrays for simulating two bosons and on the right are the waveguide arrays for simulating two fermions or two hard-core bosons.

[7,10], our results of two-particle QWs provide promising applications in exploring quantum statistics.

Furthermore, beyond a theoretical model, the two interacting quantum walkers in our models can be experimentally simulated with ultracold atoms in optical lattices and light waves in waveguides. By using spin impurities of ultracold atoms in optical lattices, two-magnon dynamics in the 1D Heisenberg $X X Z$ chain has been observed in a recent experiment [10]. It was a dramatic realization of two-HCB quantum walks with an intermediate interaction $(\Delta=|V / 2 J|=0.986)$. The strong-interaction regime $(\Delta \gg 1)$ can be achieved by Feshbach resonance [39]. Moreover, based on the quantumoptical analogs using engineered photonic waveguides [28,29], the two-particle QWs obeying the Hamiltonian (1) can be simulated via light propagations. As a single quantum walker in a $2 \mathrm{D}$ lattice is equivalent to two quantum walkers in a $1 \mathrm{D}$ lattice [3], the two-particle QWs in 1D lattices can be simulated with light waves in 2D waveguide arrays $[29,30]$. The temporal evolution of the superposition amplitude $C_{l_{1} l_{2}}$ in the twoparticle Hilbert space is mapped onto the spatial propagation of the optical field $\mathbf{E}_{l_{1} l_{2}}$ in the $\left(l_{1}, l_{2}\right)$ th waveguide. According to the evolution equation (22) of $C_{l_{1} l_{2}}$, the propagation equation for $\mathbf{E}_{l_{1} l_{2}}$ is given by

$$
\begin{aligned}
i \frac{d}{d z} \mathbf{E}_{l_{1} l_{2}}= & -J\left(\mathbf{E}_{l_{1}, l_{2}+1}+\mathbf{E}_{l_{1}, l_{2}-1}\right) \\
& -J\left(\mathbf{E}_{l_{1}+1, l_{2}}+\mathbf{E}_{l_{1}-1, l_{2}}\right)+V_{l_{1} l_{2}} \mathbf{E}_{l_{1} l_{2}},
\end{aligned}
$$

with $V_{l_{1} l_{2}}=V \delta_{l_{1}, l_{2} \pm 1}$ and the propagation distance $z$. In Fig. 5 we show the $2 \mathrm{D}$ waveguide arrays for simulating two-particle QWs with $L_{t}=21$. Similar to the $2 \mathrm{D}$ waveguide arrays used in recent experiments [29,30], the waveguide arrays shown in Fig. 5 can be fabricated in a silica substrate by direct waveguide writing with femtosecond lasers [40]. Here the interparticle interaction strength $V$ is controlled by the difference of refractive indices between green-colored and uncolored waveguides.

\section{ACKNOWLEDGMENTS}

We thank Gora Shlyapnikov for discussion. This work was supported by the National Basic Research Program of China 
under Grants No. 2012CB821305 and No. 2012CB922101, the National Natural Science Foundation of China under Grants No. 11374375 and No. 11374331, and the Ph.D. Program
Foundation of Ministry of Education of China under Grant No. 20120171110022. X.W.G. was partially supported by the Australian Research Council.
[1] Y. Aharonov, L. Davidovich, and N. Zagury, Phys. Rev. A 48, 1687 (1993).

[2] J. Kempe, Contemp. Phys. 44, 307 (2003).

[3] A. Schreiber, A. Gábris, P. P. Rohde, K. Laiho, M. Štefaňák, V. Potoček, C. Hamilton, I. Jex, and C. Silberhorn, Science 336, 55 (2012).

[4] A. M. Childs, Phys. Rev. Lett. 102, 180501 (2009).

[5] A. M. Childs, D. Gosset, and Z. Webb, Science 339, 791 (2013).

[6] T. Kitagawa, M. S. Rudner, E. Berg, and E. Demler, Phys. Rev. A 82, 033429 (2010); T. Kitagawa, E. Berg, M. Rudner, and E. Demler, Phys. Rev. B 82, 235114 (2010).

[7] T. Kitagawa, M. A. Broome, A. Fedrizzi, M. S. Rudner, E. Berg, I. Kassal, A. Aspuru-Guzik, E. Demler, and A. G. White, Nat. Commun. 3, 882 (2012).

[8] Y. E. Kraus, Y. Lahini, Z. Ringel, M. Verbin, and O. Zilberberg, Phys. Rev. Lett. 109, 106402 (2012); M. Verbin, O. Zilberberg, Y. E. Kraus, Y. Lahini, and Y. Silberberg, ibid. 110, 076403 (2013).

[9] A. Ahlbrecht, A. Alberti, D. Meschede, V. B. Scholz, A. H. Werner, and R. F. Werner, New J. Phys. 14, 073050 (2012).

[10] T. Fukuhara, P. Schauß, M. Endres, S. Hild, M. Cheneau, I. Bloch, and C. Gross, Nature (London) 502, 76 (2013).

[11] M. Karski, L. Förster, J.-M. Choi, A. Steffen, W. Alt, D. Meschede, and A. Widera, Science 325, 174 (2009).

[12] H. Schmitz, R. Matjeschk, C. Schneider, J. Glueckert, M. Enderlein, T. Huber, and T. Schaetz, Phys. Rev. Lett. 103, 090504 (2009); F. Zähringer, G. Kirchmair, R. Gerritsma, E. Solano, R. Blatt, and C. F. Roos, ibid. 104, 100503 (2010).

[13] A. Schreiber, K. N. Cassemiro, V. Potoček, A. Gábris, P. J. Mosley, E. Andersson, I. Jex, and C. Silberhorn, Phys. Rev. Lett. 104, 050502 (2010); M. A. Broome, A. Fedrizzi, B. P. Lanyon, I. Kassal, A. Aspuru-Guzik, and A. G. White, ibid. 104, 153602 (2010); A. Schreiber, K. N. Cassemiro, V. Potoček, A. Gábris, I. Jex, and C. Silberhorn, ibid. 106, 180403 (2011).

[14] T. Fukuhara, A. Kantian, M. Endres, M. Cheneau, P. Schauß, S. Hild, D. Bellem, U. Schollwöck, T. Giamarchi, C. Gross, I. Bloch, and S. Kuhr, Nat. Phys. 9, 235 (2013).

[15] J. Du, H. Li, X. Xu, M. Shi, J. Wu, X. Zhou, and R. Han, Phys. Rev. A 67, 042316 (2003).

[16] A. M. Childs, R. Cleve, E. Deotto, E. Farhi, S. Gutmann, and D. A. Spielman, Proceedings of the Thirty-Fifth Annual ACM Symposium on Theory of Computing (ACM, New York, 2003), p. 59 C68.

[17] P. L. Knight, E. Roldán, and J. E. Sipe, Phys. Rev. A 68 , 020301(R) (2003); H. B. Perets, Y. Lahini, F. Pozzi, M. Sorel, R. Morandotti, and Y. Silberberg, Phys. Rev. Lett. 100, 170506 (2008).

[18] Y. Omar, N. Paunković, L. Sheridan, and S. Bose, Phys. Rev. A 74, 042304 (2006).

[19] P. K. Pathak and G. S. Agarwal, Phys. Rev. A 75, 032351 (2007).

[20] M. Hillery, Science 329, 1477 (2010).

[21] L. Sansoni, F. Sciarrino, G. Vallone, P. Mataloni, A. Crespi, R. Ramponi, and R. Osellame, Phys. Rev. Lett. 108, 010502 (2012).
[22] A. Peruzzo, M. Lobino, J. C. F. Matthews, N. Matsuda, A. Politi, K. Poulios, X.-Q. Zhou, Y. Lahini, N. Ismail, K. Wörhoff, Y. Bromberg, Y. Silberberg, M. G. Thompson, and J. L. OBrien, Science 329, 1500 (2010); Y. Lahini, Y. Bromberg, D. N. Christodoulides, and Y. Silberberg, Phys. Rev. Lett. 105, 163905 (2010); J. D. A. Meinecke, K. Poulios, A. Politi, J. C. F. Matthews, A. Peruzzo, N. Ismail, K. Wörhoff, J. L. O'Brien, and M. G. Thompson, Phys. Rev. A 88, 012308 (2013).

[23] A. S. Solntsev, A. A. Sukhorukov, D. N. Neshev, and Y. S. Kivshar, Phys. Rev. Lett. 108, 023601 (2012).

[24] Y. Lahini, M. Verbin, S. D. Huber, Y. Bromberg, R. Pugatch, and Y. Silberberg, Phys. Rev. A 86, 011603(R) (2012).

[25] Y. Bromberg, Y. Lahini, R. Morandotti, and Y. Silberberg, Phys. Rev. Lett. 102, 253904 (2009).

[26] C. Benedetti, F. Buscemi, and P. Bordone, Phys. Rev. A 85, 042314 (2012).

[27] M. Ganahl, E. Rabel, F. H. L. Essler, and H. G. Evertz, Phys. Rev. Lett. 108, 077206 (2012); W. Liu and N. Andrei, ibid. 112, 257204 (2014).

[28] S. Longhi, Laser Photon. Rev. 3, 243 (2009).

[29] A. Szameit and S. Nolte, J. Phys. B 43, 163001 (2010).

[30] A. Szameit, I. L. Garanovich, M. Heinrich, A. A. Sukhorukov, F. Dreisow, T. Pertsch, S. Nolte, A. Tünnermann, and Y. S. Kivshar, Nat. Phys. 5, 271 (2009); G. Corrielli, A. Crespi, G. Della Valle, S. Longhi, and R. Osellame, Nat. Commun. 4, 1555 (2013).

[31] E. Farhi and S. Gutmann, Phys. Rev. A 58, 915 (1998).

[32] T. Matsubara and H. Matsuda, Prog. Theor. Phys. 16, 569 (1956).

[33] P. Jordan and E. Wigner, Z. Phys. 47, 631 (1928); J. Dziarmaga, Phys. Rev. Lett. 95, 245701 (2005).

[34] L.-M. Duan, E. Demler, and M. D. Lukin, Phys. Rev. Lett. 91, 090402 (2003); A. B. Kuklov and B. V. Svistunov, ibid. 90, 100401 (2003); J. J. García-Ripoll and J. I. Cirac, New J. Phys. 5, 76 (2003); E. Altman, W. Hofstetter, E. Demler, and M. D. Lukin, ibid. 5, 113 (2003); C. Lee, Phys. Rev. Lett. 93, 120406 (2004).

[35] A. C. Scott, J. C. Eilbeck, and H. Gilhøj, Physica D 78, 194 (1994); M. Valiente and D. Petrosyan, J. Phys. B 41, 161002 (2008); 42, 121001 (2009); J.-P. Nguenang and S. Flach, Phys. Rev. A 80, 015601 (2009).

[36] M. Takahashi, Thermodynamics of One-Dimensional Solvable Models (Cambridge University Press, Cambridge, 1999).

[37] M. Takahashi, J. Phys. C 10, 1289 (1977).

[38] K. Winkler, G. Thalhammer, F. Lang, R. Grimm, J. H. Denschlag, A. J. Daley, A. Kantian, H. P. Büchler, and P. Zoller, Nature (London) 441, 853 (2006); J. H. Denschlag and A. J. Daley, arXiv:cond-mat/0610393v1.

[39] A. Widera, O. Mandel, M. Greiner, S. Kreim, T. W. Hänsch, and I. Bloch, Phys. Rev. Lett. 92, 160406 (2004); C. Gross, T. Zibold, E. Nicklas, J. Estève, and M. K. Oberthaler, Nature (London) 464, 1165 (2010).

[40] R. R. Gattass and E. Mazur, Nat. Photon. 2, 219 (2008). 